\title{
Downregulation of cytochrome $c$ oxidase 1 induced radioresistance in esophageal squamous cell carcinoma
}

\author{
TOMOKO TAKESUE, HIROFUMI KAWAKUBO, TETSU HAYASHIDA, MAI TSUTSUI, \\ KAZUHIRO MIYAO, KAZUMASA FUKUDA, RIEKO NAKAMURA, TSUNEHIRO TAKAHASHI, \\ NORIHITO WADA, HIROYA TAKEUCHI and YUKO KITAGAWA
}

Department of Surgery, Keio University School of Medicine, Shinjuku, Tokyo 160-8582, Japan

Received October 17, 2016; Accepted July 13, 2017

DOI: $10.3892 / 01.2017 .6699$

\begin{abstract}
Comprehensive gene screening with transposons is a novel procedure for the systematic identification of resistant genes. The present study aimed to use this technique to identify candidate radioresistant genes in esophageal squamous cell carcinoma. A transposon is a base sequence that can translocate to another location in the genome at random. By inserting the cytomegalovirus promotor as a transcriptional activator in the transposon, the following gene in the new location becomes overexpressed and the gene located at the transposon insertion site is downregulated. Consequently, various transposon-tagged cells, which have differentially overexpressed or downregulated genes using the transposon method can be obtained. Following the irradiation of transposon-tagged cells, candidate radioresistant genes can be selected in order to detect the location of the transposon in the cells that have survived. A total of 11 genes were detected as candidate radioresistant genes. Cytochrome $c$ oxidase 1 (MT-CO1), an enzyme involved in apoptosis through the activation of the caspase cascade, was one of the candidate genes identified. The relative expression level of MT-COI was 0.12 in MT-CO1-downregulated cells which was significantly lower compared with the expression level in parent TE4 cells $(\mathrm{P}<0.001)$. The survival rate was $28.7 \%$ in MT-CO1-downregulated cells and $10.5 \%$ in parent TE4 cells 9 days following 5-Gy irradiation. The activity of cytochrome $c$ and caspase-3 following irradiation was significantly lower in the MT-COI-downregulated radioresistant cells compared with in TE4 cells. In conclusion, the novel gene screening technique demonstrated to be useful for detecting candidate radioresistant genes in esophageal squamous cell carcinoma. The results of the present study revealed that the downregulation of $M T-C O I$
\end{abstract}

Correspondence to: Dr Hirofumi Kawakubo, Department of Surgery, Keio University School of Medicine, 35 Shinanomachi, Shinjuku, Tokyo 160-8582, Japan

E-mail: hkawakubo@z3.keio.jp

Key words: cytochrome $c$ oxidase 1, radioresistance, esophageal cancer, transposon, gene screening induced radioresistance occurs by inhibiting the activation of the caspase cascade in radioresistant esophageal cancer cells.

\section{Introduction}

Squamous cell carcinoma (SCC) is more common in Eastern countries, including Japan. Despite the pathological difference, esophageal cancer has a poor prognosis due to early metastasis and direct invasion. Multimodal therapies including surgery, chemotherapy, and radiotherapy are necessary; however, the response rate to chemoradiation therapy is low; in one Japanese study it was found to be $68 \%$ in patients with advanced SCC in the thoracic esophagus (1). Although chemo or radiosensitivity is closely related to prognosis, it is currently impossible to predict the therapeutic effect of therapies. Therefore, detecting resistant genes and mechanisms is essential for tailoring treatment to improve the prognosis.

Many studies have shown numerous genes or microRNA related to radioresistance in esophageal cancer cells (2). A few comprehensive gene analyses by microarrays have also detected many candidate radioresistant genes $(3,4)$. However, most radioresistant genes are not yet known and the mechanisms underlying their radioresistance remain unclear.

Comprehensive gene screening with transposons is a novel procedure for systematically identifying chemoresistant genes, developed by Chen et al in 2013 (5). A transposon is a mobile genetic element which transports at random to other locations in the genome. By inserting a cytomegalovirus (CMV) promotor as a transcriptional activator in the transposon, the following gene in the new location will be overexpressed and the gene located at the transposon insertion site will be downregulated. We inserted transposons into tumor cells to form a library of 'transposon-tagged cells.' Each transposon-tagged cell has randomly activated or inactivated genes. After drug treatment is administered to the cell library, the surviving cells can either have overexpressed chemoresistant genes or downregulated chemosensitive genes due to the transposons with the CMV promoter. By detecting the location of the transposon in surviving cells, it is possible to pick up candidate chemotherapy-resistant genes. What makes this screening method distinct is that it is a gain-of-function genetic screening, unlike past genetic interrogation approaches for genomes. Thus, this screening can be used to survey untranscribed regions (5). 
Using this technique, we have already succeeded in generating cell libraries and identifying candidate genes for cisplatin resistance in esophageal squamous cell carcinoma (6). The aim of the present study is to use this method to identify candidate radioresistant genes in esophageal squamous cell carcinoma.

\section{Materials and methods}

Cell cultures and treatments. We established transposon-tagged cells in TE4 and TE15, human well-differentiated esophageal squamous cell carcinoma cell lines provided by the Cell Resource Center for Biomedical Research Institute of Development, Aging and Cancer, Tohoku University (Sendai, Japan) (6). Puromycin-resistant genes in transposons were also inserted into the transposon-tagged cells for puromycin selection. The cell lines were cultured in Dulbecco's modified Eagle's medium supplemented with $10 \%$ fetal bovine serum (Invitrogen, Carlsbad, CA, USA) and penicillin-streptomycin solution (penicillin 10,000 U/ml, streptomycin 10,000 $\mu \mathrm{g} / \mathrm{ml}$, puromycin $0.5 \mu \mathrm{g} / \mathrm{ml}$ ) (all from Nacalai Tesque, Inc., Kyoto, Japan). In all the experiments, the cells were cultured at $37^{\circ} \mathrm{C}$ in a humidified atmosphere consisting of $5 \% \mathrm{CO}_{2}$ in the air. The parent cell lines, TE4, and TE15, were cultured without puromycin.

Radiation treatment. Around 250,000 transposon-tagged cells were plated on 100-mm tissue culture dishes for radiation treatment. Dishes were irradiated by 9-11 Gy using an X-ray cell irradiator, CellRad (Faxitron Bioptics, Tucson, AZ, USA). Irradiated cells were cultured for more than 14 days until radioresistant cells (RRCs) formed colonies.

Comprehensive gene screening with transposon. The base sequences of the transposons' insertion sites were detected using Splinkerette polymerase chain reaction (PCR), TOPO cloning, and Sanger sequencing performed in accordance with the protocol of our previous study (6). Insertion sites were aligned using the BLAST function of the National Library of Medicine (http://blast.ncbi.nlm.nih. gov/Blast.cgi). We included a maximum of 15 genes that had somewhat similar sequences (blastn) as candidate genes for each colony.

Real-time quantitative PCR analysis. The cells were harvested, and total RNA was extracted with an RNeasy kit (Qiagen, Valencia, CA, USA). The concentration of total RNA was determined using a NanoDrop 2000c (NanoDrop Technologies, San Diego, CA, USA). cDNA was synthesized from total RNA using a High Capacity RNA-to-cDNA kit (Thermo Fisher Scientific, Inc., Waltham, MA, USA) in accordance with the manufacturer's protocol. PCR reaction mixes were prepared using the template cDNA samples and Fast SYBR-Green Master Mix (Thermo Fisher Scientific, Inc.), while the expressions of human cytochrome $c$ oxidase 1 (MT-CO1), cytochrome $c$ oxidase subunits 2 (MT-CO2), and 3 (MT-CO3), ND2, and GAPDH were analyzed using a ViiA7 Real-Time PCR system (Thermo Fisher Scientific, Inc). The thermal cycling reaction included incubation at $95^{\circ} \mathrm{C}$ for $20 \mathrm{sec}, 40 \mathrm{cycles}$ of $95^{\circ} \mathrm{C}$ for $3 \mathrm{sec}$, and $60^{\circ} \mathrm{C}$ for $30 \mathrm{sec}$. Data were collected using analytical software (Applied Biosystems,
Foster City, CA, USA). The expression level of each gene was determined using the $\Delta \Delta \mathrm{Ct}$ method.

MTT assay. Survival rates after irradiation were measured by an MTT assay. One day before 7-Gy irradiation, $5 \times 10^{2} /$ well of TE4 cells and MT-CO1-downregulated cells were seeded on a 96-well plate. TE4 cells and MT-CO1-downregulated cells with and without irradiation were incubated with MTT (3-(4, 5-dimethylthiazol-2-yl)-2, 5-diphenyltetrazolium bromide, a yellow tetrazole) for $3 \mathrm{~h}$ and incubated with dimethyl sulfoxide for $20 \mathrm{~min}$. The survival rate after irradiation was measured as the ratio of absorbance at $550 \mathrm{~nm}$ of irradiated cells to non-irradiated cells.

Cytochrome c oxidase assay. A cell pellet of 10 million cells was resuspended in $200 \mu \mathrm{l}$ of a cell lysis buffer containing $250 \mathrm{mM}$ sucrose, $20 \mathrm{mM}$ HEPES-KOH (pH 7.4), $10 \mathrm{mM}$ $\mathrm{KCl}, 1.5 \mathrm{mM}$ Na-EGTA, $1.5 \mathrm{mM}$ Na-EDTA, $1 \mathrm{mM} \mathrm{MgCl}_{2}$, and a protease inhibitor cocktail. After a 20-min incubation on ice, the cells were disrupted with a Dounce homogenizer (50 strokes with a loose glass pestle and 50 strokes with a tight glass pestle). The resulting homogenates were immediately used in the cytochrome $c$ oxidase assay.

Cytochrome $c$ oxidase activity was determined by using a Cytochrome $c$ Oxidase Assay kit (Sigma-Aldrich, St. Louis, MO, USA). Absorbance at $550 \mathrm{~nm}$ of ferrocytochrome $c$ was measured using NanoDrop 2000c (NanoDrop Technologies). Cytochrome $c$ oxidase activity was measured as the reduction in absorbance in the sample at $550 \mathrm{~nm}$.

To get reduced cytochrome $c, 2.7 \mathrm{mg}$ of cytochrome $c$, dissolved in $1 \mathrm{ml}$ water, was incubated with $5 \mu 1$ of $0.1 \mathrm{M}$ 1.4-dithiothreitol (DTT) for $15 \mathrm{~min}$ on ice. The reduction in absorbance at $550 \mathrm{~nm}$ for $10 \mathrm{~min}$ was measured immediately after adding $50 \mu \mathrm{l}$ of reduced cytochrome $c$ solution to $950 \mu \mathrm{l}$ of the homogenate (100 $\mu \mathrm{g}$ of total protein).

Caspase-3 activity. Caspase-3 activity was determined by using an APOPCYTO ${ }^{\mathrm{TM}}$ Caspase-3 Colorimetric Assay kit (Medical \& Biological Laboratories, Co., Ltd., Nagoya, Japan). After 5-Gy irradiation, a 3x10 $/$ well of TE4 cells and MT-CO1-downregulated cells were seeded on a 96-well plate. Caspase-3 activity was measured by NanoDrop 2000c as the absorbance at $405 \mathrm{~nm}$ after incubation with a colorimetric substrate (DEVD-pNA) with cell lysate (90 $\mu \mathrm{g}$ of total protein in $50 \mu \mathrm{l}$ of lysing buffer) at $37^{\circ} \mathrm{C}$ for $24 \mathrm{~h}$.

Statistical analysis. Univariate analyses were performed using Student's t-test or the Mann-Whitney U test. IBM SPSS Statistics version 21 (IBM Corp., Armonk, NY, USA) was used for statistical analyses. A p-value $<0.05$ was considered statistically significant.

\section{Results}

Detection of candidate radioresistant genes. After irradiation of the TE4 and TE15 transposon-tagged cells, 108 radioresistant colonies were picked up. The nucleotide sequences of the transposons' insertion sites in each colony were amplified by Splinkerette PCR and TOPO cloning. Fifty nucleotide 
Table I. List of the 11 candidate radioresistant genes detected by cyclopedic gene screening with transposons.

Gene

Overexpression/downregulation Number of detection

Sorting nexin 3 (SNX3)

Cytochrome $c$ oxidase 1 (MT-CO1)

Mediator of RNA polymerase II transcription subunit 13 (MED13)

Yae1 domain containing protein 1 isoform 2 (YAE1D1)

Centromere protein $\mathrm{V}$

Signal-induced proliferation associated 1 like protein2 (SIPA1L2)

Beta crystallin A4 (CRYBA4)

RNA-binding motif single-stranded interacting protein 3 (RBMS3)

Transcription factor 4 isoform 1 , isoform 4

Protein FAM178B isoform A (FAM178B)

Nck-associated protein 5 (NCKAP5)

$\begin{array}{ll}\text { Downregulation } & 3 \\ \text { Downregulation } & 3 \\ \text { Downregulation } & 2 \\ \text { Overexpression } & 1 \\ \text { Downregulation } & 1 \\ \text { Downregulation } & 1 \\ \text { Overexpression } & 1 \\ \text { Downregulation } & 1 \\ \text { Downregulation } & 1 \\ \text { Downregulation } & 1 \\ \text { Downregulation } & 1\end{array}$

sequences were thus, detected. Insertion sites were searched for by aligning the detected nucleotide sequences on the BLAST site. The genes located up to $25 \mathrm{kbp}$ downstream from the insertion site were identified as candidate radioresistant genes. Eleven such genes were detected; these are listed in Table I.

Nucleotide sequences of the transposon's insertion site matched a part of the gene sequence of nine genes which may have been downregulated by the inserted transposons. Two genes were detected downstream of the transposon's insertion site and may have been overexpressed by the CMV promoter in the transposon. Three genes were detected in two or three different radioresistant colonies.

MT-COl was one of the detected candidate radioresistant genes, found in three radioresistant colonies. One hundred ninety-one nucleotide bases, detected by sequence, matched the base sequence of $M T-C O 1$ coded in mitochondrial DNA (mtDNA) with a 99\% concordance rate. Cytochrome $c$ oxidase is known to be an enzyme associated with apoptosis by activating the caspase cascade. Thus, we conducted further experiments on the hypothesis that downregulation of $M T-C O 1$ induced radioresistance by blocking activation of the caspase cascade in esophageal cancer cells.

Downregulation of MT-COI in RRCs. In RRCs in which transposon was inserted into the MT-CO1, MT-COl may be downregulated by the inserted transposon; and $M T-C O 2$ and $M T-C O 3$, coded downstream of $M T-C O 1$, could possibly have been overexpressed by the CMV promotor in the transposon (Fig. 1A). The relative expression levels of $M T-C O 1, M T-C O 2$, and $M T-C O 3$ were $0.12,0.90$, and 1.15 , respectively, when the expression level of parent TE4 cell was 1. MT-CO1 was significantly downregulated in these cells $(\mathrm{P}<0.001)$ (Fig. 1B).

Radioresistance and downregulation of MT-CO1. To establish whether the RRCs (in which MT-CO1 was inactivated) showed radioresistance, survival rates after irradiation were measured by an MTT assay (Fig. 2). The relative survival rate became significantly higher compared to non-irradiated cells in the MT-CO1-downregulated cells 5 days after $7-G y$ irradiation $(\mathrm{P}<0.001)$. Although the survival
$A$

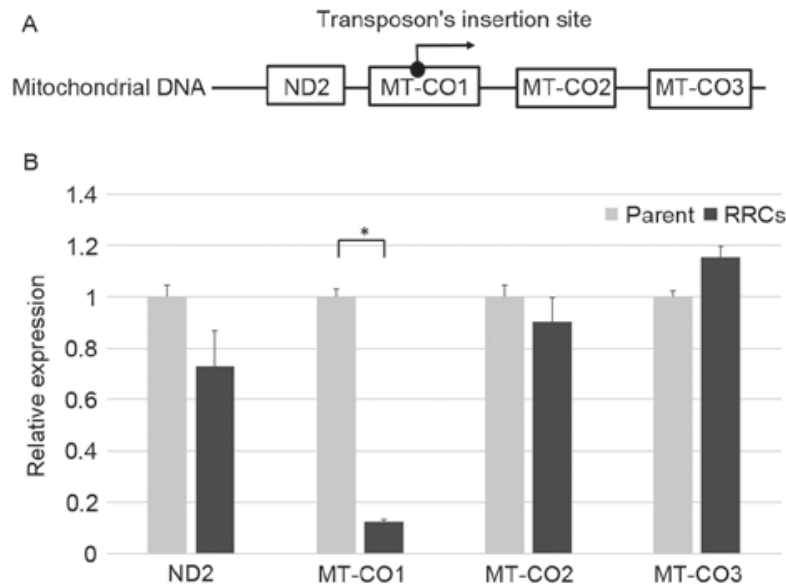

Figure 1. Downregulation of $M T$-CO1. (A) The detected base sequence was matched with a part of the $M T-C O 1$ gene sequence. If a transposon is inserted into the $M T$ - $C O 1$ gene, $M T-C O 1$ may be downregulated and the following gene (MT-CO2 or $M T$-CO3) may be overexpressed. (B) The expression level of $M T-C O 1$ was 3.4 in the RRCs, in which the transposon had transported into the $M T-C O 1$ gene, and 28.0 in the parent TE4 cells. There was a significant difference between the two groups $(\mathrm{P}<0.001)$. ND2, $M T-C O 2$, and $M T-C O 3$ were not overexpressed in the RRCs. (Light bars, parent cells; dark bars, RRCs). Error bars represent the standard deviation of the mean. MT-CO1, cytochrome $c$ oxidase 1; RRCs, radioresistant cells.

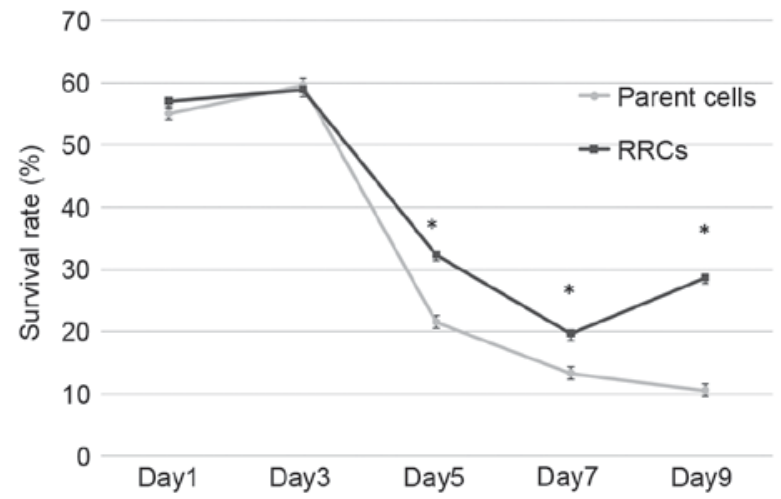

Figure 2. Radioresistance in the cells with downregulation of MT-CO1. Cell survival rates were measured at $1,3,5,7$, and 9 days after 7-Gy irradiation. The survival rates of $M T$-CO1-downregulated cells were significantly higher in days 5, 7 and $9(\mathrm{P}<0.001)$. Error bars represent the standard deviation of the mean. MT-CO1, cytochrome $c$ oxidase 1; RRCs, radioresistant cells. 


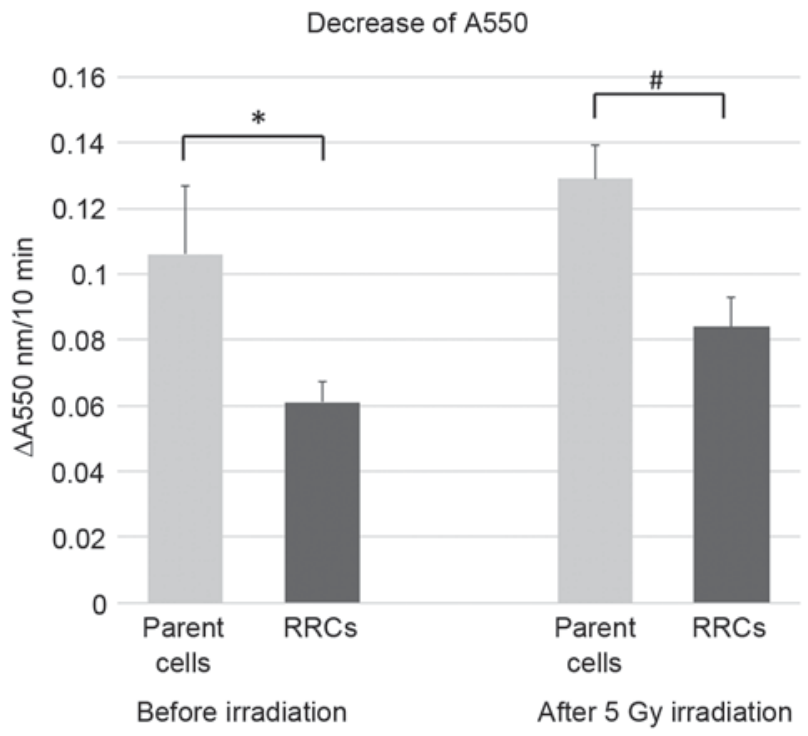

Figure 3. Cytochrome $c$ oxidase assay. The activity of cytochrome $c$ oxidase was measured as the reduction in absorbance at $550 \mathrm{~nm}$, a characteristic wavelength for ferrocytochrome $c$. Cytochrome $c$ oxidase activity was significantly reduced because of $M T-C O 1$ downregulation in the RRCs $\left({ }^{*} \mathrm{P}=0.023\right)$. Although cytochrome $c$ oxidase activity was increased in both the parent and RRCs after 5-Gy irradiation, it was significantly lower in the RRCs ( $\left.{ }^{\#} \mathrm{P}=0.005\right)$. Error bars represent the standard deviation of the mean. MT-CO1, cytochrome $c$ oxidase 1; RRCs, radioresistant cells.

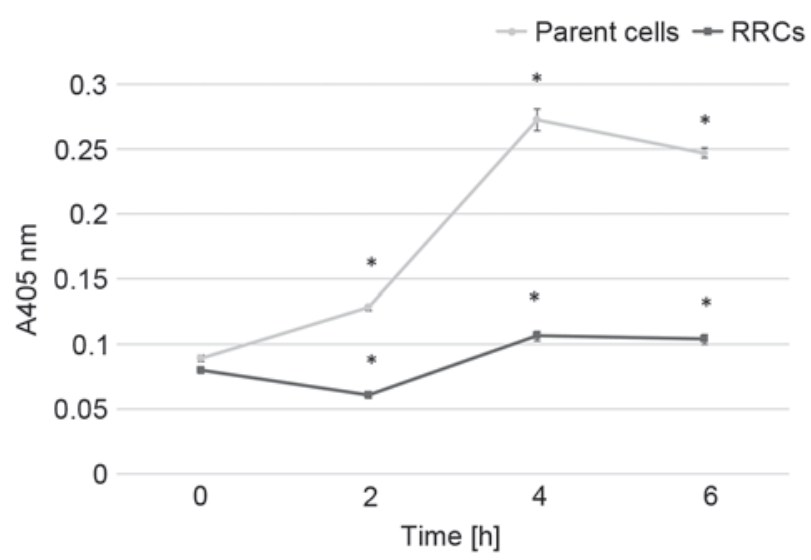

Figure 4. Caspase-3 activity after irradiation. After 5-Gy irradiation, the parent (TE4) cells and RRCs (MT-CO1-downregulated cells) were incubated with colorimetric substrate DEVD-pNA for $24 \mathrm{~h}$. Caspase-3 activity was measured as the absorbance at $405 \mathrm{~nm}$ and was found to be higher in parent cells after irradiation $\left({ }^{*} \mathrm{P}<0.001\right)$. Error bars represent the standard deviation of the mean. MT-CO1, cytochrome $c$ oxidase 1; RRCs, radioresistant cells.

rate kept decreasing in the parent (TE4) cells, it increased in the RRCs (MT-CO1-downregulated cells) at day 9. The difference between the parent cells and RRCs increased, with survival rates at day 9 of 10.5 and $28.7 \%$, respectively $(\mathrm{P}<0.001)$.

The activation of cytochrome coxidase was inhibited in RRCs. The cytochrome $c$ oxidase changed reduced cytochrome $c$ (ferrochrome $c$ ) into oxidized cytochrome $c$ (ferrichrome $c$ ). Cytochrome $c$ has a sharp absorption band at $550 \mathrm{~nm}$ in the reduced state. Activation of cytochrome $c$ oxidase was measured as a decrease in absorbance at $550 \mathrm{~nm}$ after adding cytochrome $c$ reduced by DTT.
Fig. 3 shows the decrease in the absorbance at $550 \mathrm{~nm}$, which was 0.106 in the parent (TE4) cells and 0.061 in the RRCs before irradiation. Six h after 5-Gy irradiation, the decrease in absorbance was 0.129 in the parent cells and 0.084 in the RRCs. Activation of cytochrome $c$ oxidase was significantly lower in RRCs with MT-CO1 downregulation both before and after irradiation $(\mathrm{P}=0.023$ and $\mathrm{P}=0.005$, respectively).

The activation of caspase-3 was inhibited in RRCs after irradiation. To show that activation of the caspase cascade induced by oxidized cytochrome $c$ was suppressed in RRCs after irradiation, we measured the activity of caspase-3.

Caspase-3 activity after 5-Gy irradiation is shown in Fig. 4. In the parent cells, caspase-3 activity rapidly increased after irradiation and reached its peak at $4 \mathrm{~h}$ after the irradiation. Caspase- 3 activity gradually increased after irradiation in the RRCs, although it remained significantly lower than in the parent cells $(\mathrm{P}<0.001)$.

\section{Discussion}

We detected $M T-C O 1$ as a candidate radioresistant gene from radioresistant transposon-tagged esophageal squamous cancer cells. Downregulation of $\mathrm{MT}$-COI was verified by real-time quantitative PCR analysis of RRCs. RRCs with the downregulation of MT-COI showed higher survival rates than parent cells after irradiation. We also showed that inhibiting apoptosis by the activation of caspase cascade caused radioresistance because the activity of cytochrome $c$ and caspase-3 after irradiation was significantly lower in the $M T$-COl-downregulated RRCs.

$M T$-CO, or complex IV, is the terminal complex of the electron transport chain on the mitochondrial membrane. It is composed of three catalytic subunits encoded in mtDNA (subunits 1 to 3 ) and 10 accessory subunits (subunits 4 to 13) encoded in nuclear DNA $(7,8)$.

It is known that cytochrome $c$ oxidase is related to apoptosis (9). Cytochrome $c$, released from mitochondria into the cytosol by various apoptotic stimuli, binds to Apaf-1 and forms the apoptosome which, in turn, activates pro-caspase- 9 leading to apoptosis (10). The redox state of cytochrome $c$ is important in this caspase-dependent apoptosis. Only oxidized cytochrome $c$ can activate the apoptosome, whereas reduced cytochrome $c$ cannot (11-13). The most potent enzyme that oxidizes cytochrome $c$ is cytochrome $c$ oxidase (13). Multiple caspase-dependent and -independent cell death pathways induce apoptosis after DNA damage (14). Our results revealed that downregulation of the MT-CO1 gene could not activate casepase- 3 in a few hours after irradiation. It seemed to affect radioresistant, high survival rate in 5 to 9 days after irradiation, in our study.

The relationship between cytochrome $c$ oxidase and chemo or radioresistance has been reported in various tumor cell lines (15-19). Comprehensive gene analysis on a microarray in cervical carcinoma cells (squamous cell carcinoma) showed that $M T-C O 1$ was increased by a factor greater than two in RRCs compared with radiosensitive cells (15). Also, in human acute myelogenic leukemia cells, MT-CO3 was upregulated in chemoresistant cells compared with in chemosensitive 
cells (16). However, these study did not verify that overexpression of MT-COI actually induces radioresistance. Because the technique for transfection into mitochondrial DNA has not yet been established, it is impossible to overexpress or downregulate a mitochondrial gene (17). Aichler et al (18) reported that knockdown of MT-CO7A2 with siRNA increased chemosensitivity after chemotherapy with cisplatin and 5-flurouracil in esophageal adenocarcinoma cells. Oliva et al $(19,20)$ revealed that pharmacologic and genetic manipulation of cytochrome $c$ oxidase restored chemosensitivity in chemoresistant glioma cells. From these and our results, cytochrome $c$ oxidase seems to be a significant gene which induces chemo or radioresistance, and it may be a target gene for treatment to improve chemo or radiosensitivity.

A question raised by our study is about the homogeneity of mtDNA. Each cell contains hundreds to thousands of copies of mtDNA, so if a transposon transports into the $M T-C O 1$ gene in one mtDNA, it should not affect the properties of the whole cell. However, in this study MT-COI was actually downregulated in RRCs. It is known that, in general, all copies of mtDNA are identical within a cell, a genetic state known as homoplasmy (21). In cell proliferation, only one mtDNA is copied by rolling circle DNA replication (22). Although this mechanism has not yet been completely clarified, homoplasmy of mtDNA could relate to the downregulation of MT-COI.

It was unclear whether $M T-C O 1$ gene mutation was actually related to radioresistance in patients with esophageal cancer. Multiple gene mutations with complicated interactions could induce radioresistance. Although our comprehensive gene screening is ongoing, it is proving to be a useful approach for detecting radioresistant genes. Verification of the relationships between all the detected radioresistant genes and the clinical responses to radiation therapy is essential for clinical application.

In conclusion, eleven candidate radioresistant genes in esophageal squamous cancer were detected by comprehensive gene screening with transposons. The mechanism for radioresistance induced by $M T-C O 1$ downregulation was also revealed. The candidate genes were detected under conditions more similar to a clinical setting than was the case in previous forms of gene screening. By detecting all radioresistant genes and revealing the underlying mechanisms for this, we can select the appropriate therapy for patients or enhance the effect of the therapy by treating target genes.

\section{References}

1. Ishida K, Ando N, Yamamoto S, Ide H and Shinoda M: Phase II study of cisplatin and 5-fluorouracil with concurrent radiotherapy in advanced squamous cell carcinoma of the esophagus: A Japan Esophageal Oncology Group (JEOG)/Japan Clinical Oncology Group trial (JCOG9516). Jpn J Clin Oncol 34: 615-619, 2004.

2. Guo W and Jiang YG: Current gene expression studies in esophageal carcinoma. Curr Genomics 10: 534-539, 2009.

3. Fukuda K, Sakakura C, Miyagawa K, Kuriu Y, Kin S, Nakase Y, Hagiwara A, Mitsufuji S, Okazaki Y, Hayashizaki Y and Yamagishi H: Differential gene expression profiles of radioresistant oesophageal cancer cell lines established by continuous fractionated irradiation. Br J Cancer 91: 1543-1550, 2004.
4. Ogawa R, Ishiguro H, Kuwabara Y, Kimura M, Mitsui A, Mori Y, Mori R, Tomoda K, Katada T, Harada K and Fujii Y: Identification of candidate genes involved in the radiosensitivity of esophageal cancer cells by microarray analysis. Dis Esophagus 21: 288-297, 2008.

5. Chen L, Stuart L, Ohsumi TK, Burgess S, Varshney GK, Dastur A, Borowsky M, Benes C, Lacy-Hulbert A and Schmidt EV: Transposon activation mutagenesis as a screening tool for identifying resistance to cancer therapeutics. BMC Cancer 13: 93, 2013.

6. Tsutsui M, Kawakubo H, Hayashida T, Fukuda K, Nakamura R, Takahashi T, Wada N, Saikawa Y, Omori T, Takeuchi H and Kitagawa Y: Comprehensive screening of genes resistant to an anticancer drug in esophageal squamous cell carcinoma. Int J Oncol 47: 867-874, 2015.

7. Kadenbach B, Jarausch J, Hartmann R and Merle P: Separation of mammalian cytochrome $c$ oxidase into 13 polypeptides by a sodium dodecyl sulfate-gel electrophoretic procedure. Anal Biochem 129: 517-521, 1983.

8. Weishaupt A and Kadenbach B: Selective removal of subunit VIb increases the activity of cytochrome $c$ oxidase. Biochemistry 31: 11477-11481, 1992.

9. Pradelli LA, Bénéteau M and Ricci JE: Mitochondrial control of caspase-dependent and -independent cell death. Cell Mol Life Sci 67: 1589-1597, 2010.

10. Brown GC and Borutaite V: Regulation of apoptosis by the redox state of cytochrome $c$. Biochim Biophys Acta 1777: 877-881, 2008.

11. Pan Z, Voehringer DW and Meyn RE: Analysis of redox regulation of cytochrome $c$-induced apoptosis in a cell-free system. Cell Death Differ 6: 683-688, 1999.

12. Suto D, Sato K, Ohba Y, Yoshimura T and Fujii J: Suppression of the pro-apoptotic function of cytochrome $c$ by singlet oxygen via a haem redox state-independent mechanism. Biochem J 392: 399-406, 2005.

13. Borutaite V and Brown GC: Mitochondrial regulation of caspase activation by cytochrome oxidase and tetramethylphenylenediamine via cytosolic cytochrome $c$ redox state. J Biol Chem 282: 31124-31130, 2007.

14. Kim R, Emi M and Tanabe K: Caspase-dependent and -independent cell death pathways after DNA damage (Review). Oncol Rep 14: 595-599, 2005.

15. Achary MP, Jaggernauth W, Gross E, Alfieri A, Klinger HP and Vikram B: Cell lines from the same cervical carcinoma but with different radiosensitivities exhibit different cDNA microarray patterns of gene expression. Cytogenet Cell Genet 91: 39-43, 2000.

16. Huang TS, Myklebust LM, Kjarland E, Gjertsen BT, Pendino F, Bruserud Ø, Døskeland SO and Lillehaug JR: LEDGF/p75 has increased expression in blasts from chemotherapy-resistant human acute myelogenic leukemia patients and protects leukemia cells from apoptosis in vitro. Mol Cancer 6: 31, 2007.

17. Inoue K, Nakada K, Ogura A, Isobe K, Goto Y, Nonaka I and Hayashi JI: Generation of mice with mitochondrial dysfunction by introducing mouse mtDNA carrying a deletion into zygotes. Nat Genet 26: 176-181, 2000.

18. Aichler M, Elsner M, Ludyga N, Feuchtinger A, Zangen V, Maier SK, Balluff B, Schöne C, Hierber L, Braselmann H, et al: Clinical response to chemotherapy in oesophageal adenocarcinoma patients is linked to defects in mitochondria. J Pathol 230: 410-419, 2013.

19. Oliva CR, Nozell SE, Diers A, McClugage SG III, Sarkaria JN, Markert JM, Darley-Usmar VM, Bailey SM, Gillespie GY, Landar A and Griguer CE: Acquisition of temozolomide chemoresistance in gliomas leads to remodeling of mitochondrial electron transport chain. J Biol Chem 285: 39759-39767, 2010.

20. Oliva CR, Moellering DR, Gillespie GY and Griguer CE: Acquisition of chemoresistance in gliomas is associated with increased mitochondrial coupling and decreased ROS production. PLoS One 6: e24665, 2011.

21. Lightowlers RN, Chinnery PF, Turnbull DM and Howell N: Mammalian mitochondrial genetics: Heredity, heteroplasmy and disease. Trends Genet 13: 450-455, 1997.

22. Shibata T and Ling F: DNA recombination protein-dependent mechanism of homoplasmy and its proposed functions. Mitochondrion 7: 17-23, 2007. 